\title{
HERZINFARKT
}

\section{Erste Warnzeichen im Bett}

Erektionsschwäche kann ein Vorbote eines Herzinfarkts sein. Darauf deutet eine Studie der Universitätsklinik von Kuala Lumpur, Malaysia, hin. Dreiviertel von 111 sexuell aktiven Patienten, die mit einem transmuralen Herzinfarkt eingeliefert wurden, berichteten über Potenzprobleme in den sechs Monaten vor dem Infarkt. $25 \%$ von 81 sexuell inaktiven Infarktpatienten waren schon länger komplett erektionsunfähig. KHK und erektile Dysfunktion (ED) haben gemeinsame Risikofaktoren, insbesonde- re die Atherosklerose. Da die Penisarterien kleinkalibriger sind als die Koronararterien, manifestiert sich die ED häufig vor der KHK, berichtete Studienautor Dr. S.V. Ramesh.

Sein Rat: „Wir sollten Patienten mit Erektionsproblemen als Hochrisikopatienten für einen Herzinfarkt ansehen." Auch bei Männern, die ein Sexualleben verneinen, empfiehlt er eine kardiologische Abklärung.

World Congress of Cardiology, Peking, Juni 2010

\section{SCHLAGANFALL}

\section{Die wichtigsten Risikofaktoren}

Zehn Risikofaktoren sind für $90 \%$ aller Schlaganfälle verantwortlich. Das ist das Ergebnis der INTERSTROKE-Studie, in der 3000 Patienten mit einem ersten Schlaganfall (ischämisch oder hämorrhagisch) und 3000 Kontrollpersonen ohne Schlaganfall aus 22 Ländern gegenübergestellt wurden. Bereits fünf dieser Risikofaktoren erklären $80 \%$ aller Insulte. Diese sind:

(1) Bluthochdruck,

\begin{abstract}
(2) Rauchen,
(3) abdominelle Adipositas,

(4) ungesunde Ernährung und

(5) zu wenig Bewegung.

Werden fünf weitere Risikofaktoren hinzugenommen (Apo B/A1 erhöht, Diabetes mellitus, Alkoholkonsum, Stress und Depressionen), erfasst man sogar $90 \%$ des Schlaganfallrisikos in der Bevölkerung. World Congress of Cardiology, Peking, Juni 2010
\end{abstract}

\section{ANTIPHLOGISTISCHE LANGZEITTHERAPIE}

\section{Coxib besser als NSAR plus PPI}

Herkömmliche Antiphlogistika unter Protonenpumpenhemmer-Schutz oder ein selektiver COX-2-Hemmer: Was ist die bessere Wahl für einen Patienten mit Arthrose oder rheumatoider Arthritis? Bekannt ist, dass beide Optionen gleich gut vor Komplikationen im oberen Gastrointestinaltrakt schützen. Ob sie im gesamten GI-Trakt eine vergleichbare Schutzwirkung aufweisen, wurde jetzt in der CONDOR-Studie untersucht. 4484 Patienten mit erhöhtem GI-Risiko nahmen sechs Monate lang täglich entweder $2 \times 200 \mathrm{mg}$ Celecoxib oder $2 \times 75 \mathrm{mg}$ Diclofenac retard plus 1 x $20 \mathrm{mg}$ Omeprazol ein.
Zu Gl-Komplikationen kam es bei 20 Patienten (0,9\%) in der Coxibgruppe und bei 81 $(3,8 \%)$ in der Vergleichsgruppe $(p<0,0001)$. Für den Unterschied verantwortlich waren v. a. klinisch relevante Anämien aufgrund von GI-Blutungen. 6\% der Patienten unter Celecoxib und $8 \%$ unter Diclofenac/Omeprazol brachen die Therapie wegen GI-Nebenwirkungen $a b$. In der Praxis dürfte der Vorteil zugunsten des Coxibs noch deutlicher ausfallen, weil die Patienten erfahrungsgemäß früher oder später den Säureblocker weglassen, sagte Studienleiter Prof. Dr. H. Kellner aus München.

The Lancet, early online publication 17. Juni 2010

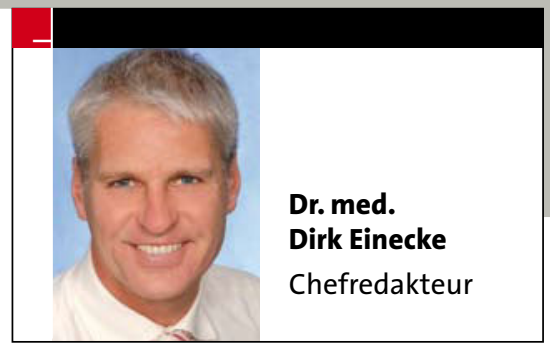

\section{SEXSOMNIE}

\section{Verbreitetes „Laster“ bei Schlafstörungen}

Wer schläft, sündigt nicht? Von wegen. Eine kanadische Studie fand bei Patienten, die sich zur Abklärung von Schlafstörungen in ein Schlafzentrum begeben hatten, überraschend häufig eine bizarre Störung, die als „Sexsomnie“ bezeichnet wird. Die Betroffenen nehmen im Schlaf an sich selbst oder an ihrem Bettpartner sexuelle Handlungen vor. Diese können von der Masturbation bis zum gewaltsamen Geschlechtsverkehr reichen. In der Studie waren Männer mit 11\% fast dreimal so häufig „nachtaktiv“ wie Frauen.

SLEEP 2010, San Antonio/Texas, 7. Juni 2010

\section{SAUERSTOFF BEI HERZINFARKT}

\section{Mehr Schaden als Nutzen?}

Die Gabe von Sauerstoff bei Herzinfarkt ist möglicherweise nicht nur von geringem Nutzen, sondern könnte sogar schädlich sein. Das legt eine Cochrane-Metaanalyse nahe, in der drei randomisierte Studien mit 387 Infarktpatienten ausgewertet wurden. Insgesamt ereigneten sich 14 Todesfälle. Der Einsatz von Sauerstoff war mit einem relativen Mortalitätsrisiko von 3,03 assoziiert. Angesichts der geringen Patientenzahlen könnte es sich um einen zufälligen Effekt handeln. Der Zusammenhang muss jedoch umgehend in einer randomisierten Studie geprüft werden, fordern die Studienautoren.

Cabello JB et al. Cochrane Database of Systematic Reviews 2010 